\title{
Caracterización Macroscópica De Morteros Romanos De Tipo Industrial En Villamanta (Madrid). Primeros Datos
}

\author{
Pablo Guerra García, (PhD) \\ Universidad Politécnica de Madrid, Spain \\ Isabel Marqués Martín, (Bachelor) \\ I.M. Arqueología y Patrimonio, Spain \\ Alejandro Bermúdez Medel, (PhD) \\ Universidad de Valladolid, Spain \\ Alberto Polo Romero, (PhD) \\ Universidad Rey Juan Carlos, Spain
}

Doi: 10.19044/esj.2017.v13n35p1 URL:http://dx.doi.org/10.19044/esj.2017.v13n35p1

\begin{abstract}
The objective of this article is to show the results obtained from the physical and macroscopic characterization of the mortars sampled by Isabel Marqués and Alejandro Bermúdez, archaeological directors of the intervention carried out in El Tejar settlement, in the municipality of Villamanta (Madrid, Spain). The results of this work have determined a relevant typological classification among the samples, highlighting the presence of siliceous aggregates, ceramics in some mortars, a very varied grading and a distribution of the components that have conditioned the conservation. Of the four samples taken -VMT1 to VMT4- one of them has an aqueous pigmentation, belonging to an inside plaster, while the others correspond to lime mortars with aggregates from hydraulic surfaces.

The archaeological investigation of the deposit of Villamanta is complemented by an implementation check of lime mortars, being one of the most important Roman context of Madrid. This research add a sequence of new results around the Roman mortars and implements a methodology in the study of the historical mortars.
\end{abstract}

Keywords: Mortar, characterization, binder, Villamanta, archaeology

\section{Resumen}

El presente artículo tiene como objetivo mostrar los resultados obtenidos de la caracterización física y macroscópica de las muestras de 
mortero recogidas por Isabel Marqués y Alejandro Bermúdez, directores arqueólogos de la intervención llevada a cabo en el yacimiento de El Tejar, en el término municipal de Villamanta (Madrid).

Los resultados de este trabajo han determinado una clasificación tipológica relevante entre las muestras, destacando la presencia de áridos silíceos, cerámicos en algunos morteros, una granulometría muy variada y una distribución de los componentes que ha condicionado su estado de conservación. De las cuatro muestras tomadas -VMT1 a VMT4- una de ellas presenta una pigmentación acuosa, perteneciendo a algún enlucido interior, mientras que las restantes corresponden a morteros de cal con áridos propios de superficies hidráulicas.

La realización de este estudio complementa la investigación arqueológica del yacimiento de Villamanta, uno de los más importantes del contexto romano en la Comunidad de Madrid, incorpora nuevos datos referidos a los morteros romanos e implementa una metodología de estudio de las argamasas históricas, importantes en el conocimiento de los morteros históricos.

Palabras-clave: Mortero, caracterización, áridos, Villamanta, arqueología

\section{Introducción}

De los estudios y documentación disponibles se desprende que Villamanta, en época romana, fue un núcleo poblacional de cierta relevancia en cuyo entorno próximo, utilizando como eje la vega del Arroyo Grande, se desarrolló una activa vida, que se supone de carácter agropecuario e industrial. Debió estar vinculado a una vía de comunicación y provisto de una o varias áreas cementeriales. Su identificación con Mantua Carpetanorum es más que probable, aunque se halle sujeta a discusión (Ptolomeo, II, 6,56; López, 1788; Pellicer, 1803; Montero, 1990; Stylow, 1990; Gamallo y Gimeno, 1990; Alvar, 1993; Contreras et al., 1993; TIR 1993; Gómez-Reino et al., 1994; Oñate, 2001a y 2001b; Zarzalejos, 2002 Herrera y Velasco, 2003; Urbano, 2005 y 2010; Martínez, 2006 y 2007; Fernández, 2007, 2008 y 2010; Carta Arqueológica de la Comunidad de Madrid; Arkatros, 2011; Marqués, Bermúdez y Hoces de la Guardia, 2016, Hoces de la Guardia, Bermúdez y Marqués, 2015 y Bermúdez y Marqués, 2016).

El área sometida a nuestra intervención en los años 2014 y 2015 es un terreno en la vega del Arroyo Grande (margen izquierda) que forma un amplio valle abierto, antaño inundable en la época de avenidas, delimitado al sur por suaves lomas, al pie de cuya pendiente se encuentra precisamente el yacimiento que ha resultado de entidad: El Tejar.

Se han localizado diversas estructuras y elementos singulares de carácter antrópico. Las halladas en el sector 5 pudieron pertenecer a un 
pequeño torcularium, donde se produciría el prensado de la uva para producir mosto; o aceite, si lo relacionamos con un topónimo próximo: Los Olivos.

La UE 506 podría formar parte del calcatorium (zona de prensado), mientras que la UE 503, sería el lacus (depósito del líquido extraído). Nada sabemos sobre el resto de elementos que constituirían la instalación completa (Gómez Delgado, s.f.; Marqués, 2007). De aquí proceden las muestras de mortero recogidas y analizadas. Este hipotético torcularium, podría ser de uso interno de una villa o establecimiento rural.

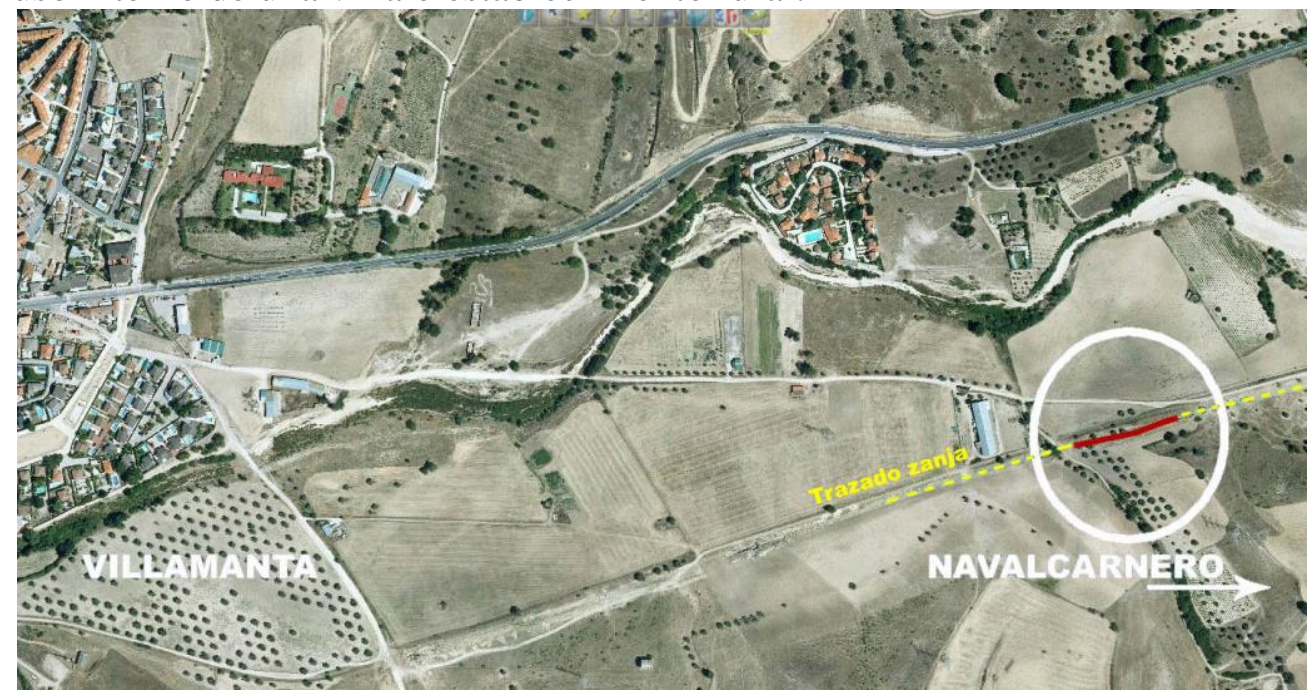

Fig. 1. Panorámica aérea de la zona arqueológica intervenida.

En general, los restos documentados permiten afirmar que nos encontramos ante una parte de una instalación agropecuaria muy próxima al río y, por su carácter, dependiente de un complejo de mayor entidad situado en las lomas que se elevan suavemente en dirección sur, a salvo de inundaciones ocasionales. Por su proximidad al curso de agua principal, pero sobre todo por su inmediatez a pequeños arroyos que descendían de las tierras circundantes, debió ser protegida con un básico sistema de drenaje que lateralmente recogería las aguas desbordadas durante las avenidas, encauzándolas hasta lugar seguro. Consideramos un arco cronológico amplio de ocupación de El Tejar entre el siglo II y el IV y con un auge de actividad entre fines del II y el III. 


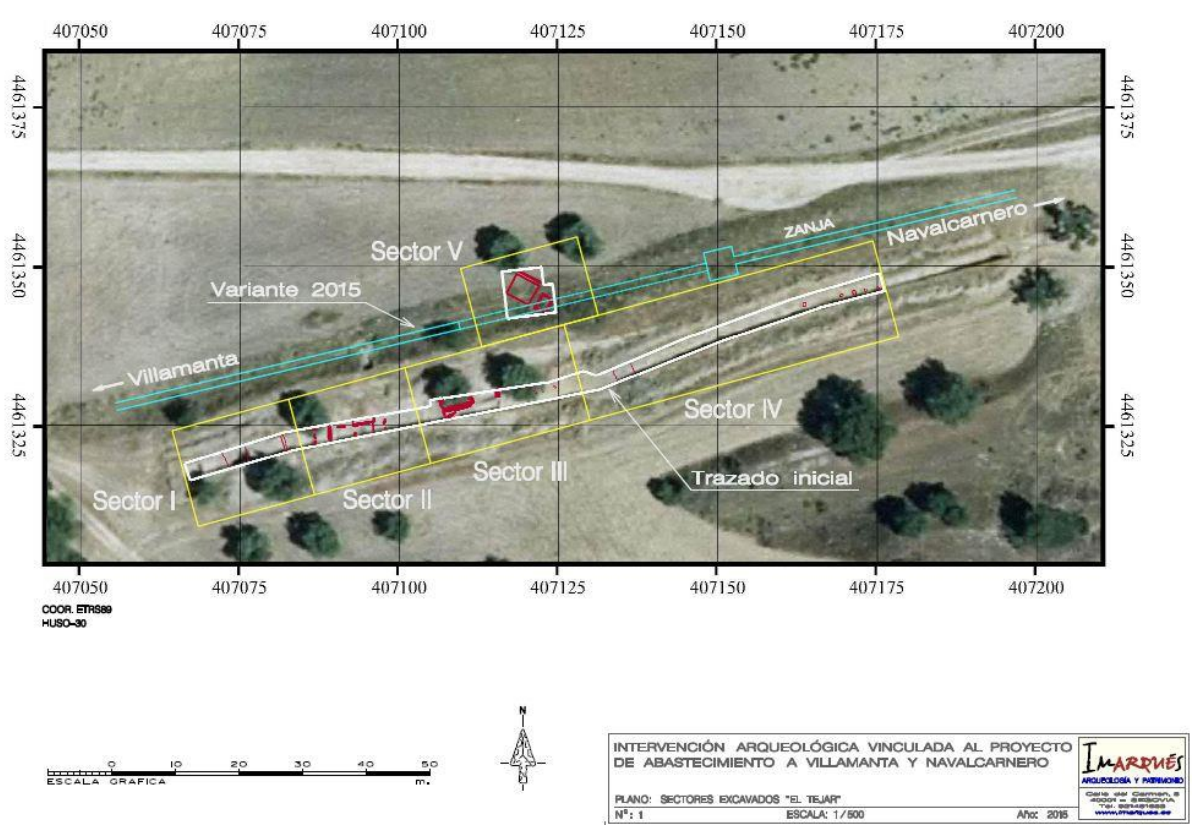

Fig. 2. Localización de los sectores en los que se distribuyen las estructuras analizadas.

Una importante característica diferencial de El Tejar es la relevante presencia de material epigráfico reaprovechado, si bien, el muro UE 324, donde se han localizado seis fragmentos de estelas funerarias, debería considerarse en este yacimiento y en el entorno inmediato, un unicum mientras no se demuestre lo contrario (las piezas que se han hallado en él suponen ahora el $40 \%$ de las estelas funerarias documentas en Villamanta).

Una vez que finalizaron las labores de documentación arqueológica del yacimiento se determinó la importancia de poder analizar las argamasas, con el objetivo de establecer unas diferencias formales, materiales y constructivas. Dichas diferencias no marcan una cronología absoluta entre las muestras sino que establecen unos patrones constructivos que pueden combinarse con los datos arqueológicos, los cuales sí establecen una cronología más fiable. Este proceso se ha seguido en otros trabajos consultados, con buenos resultados en todos ellos (Miriello et al., 2011).

\section{Metodología de estudio}

La caracterización y el estudio de los morteros históricos cuenta con numerosos precedentes. Desde los primeros trabajos de ensayo, llevados a cabo por Jedrzejewska hasta los sistemáticos de Elsen, se han ido incorporando nuevas técnicas y metodologías como la petrografía, que perfeccionan y mejoran los resultados en el conocimiento de las argamasas históricas (Jedrzejewska, 1960; Gülec y Tulun, 1997; Elsen, 2005). 
Siguiendo la secuencia metodológica, la toma de las muestras se llevó a cabo siguiendo estrictamente los protocolos de muestreo establecidos por las instituciones internacionales como el RILEM, así como por las referencias bibliográficas a las que se ha acudido (Bartos et al. 2000; Hugues y Callebaut, 2000). Las muestras fueron tomadas por el arqueólogo técnico Alberto Polo Romero, previo análisis de las estructuras por parte de los directores arqueólogos.

El muestreo se llevó a cabo con instrumental mecánico, sin el uso de productos químicos y llevando a cabo una completa documentación fotográfica del proceso. En palabras de Patricia Adriano (Adriano et al., 2009: 611):

The sampling of mortars is a crucial step that can influence the success of the characterisation methodology.

Una vez terminada la extracción las muestras fueron embolsadas y sigladas. Cada muestra no superó los 100 gramos de peso, y por lo tanto, la alteración en las fábricas originales ha sido mínima. Ya en el laboratorio se procedió a realizar una primera caracterización de visu, en el estado original de las muestras y sin ningún tratamiento, siguiendo el mismo procedimiento que en otros trabajos consultados (Broekmans Maarten, 2009; Guleç y Tulun, 1997). La macroscopía se realizó previo lavado de las muestras, primero en seco, y después con agua destilada. Puesto que alguna muestra presentaba indicios de pigmentaciones, éstas solo se limpiaron con un cepillo de cerda fina y sin aplicar líquido de ningún tipo.

La macroscopía está basada en los principios establecidos por Jeremy Ingham en cuanto a la distribución y dispersión de los áridos y gravas (Ingham, 2010), y que se pueden encontrar en otros trabajos de similares características a éste (Boscheti et al., 2008; Mertens et al., 2009). En el presente estudio se llevó a cabo tanto la identificación de los componentes y su forma, según la clasificación Raymond, como el estudio granulométrico y microestratigráfico de los morteros. Para clasificar la distribución de los componentes se ha empleado un sistema de "vectorización" que alinea los áridos y configura una red en función de su colocación en la sección. Este sistema se ha llevado a la práctica en una tesis doctoral inédita (Guerra García, 2015).

En dicha tesis se ha desarrollado un procedimiento de análisis macroscópico, microscópico y químico que ha determinado la naturaleza de los anillos de reacción alrededor de los áridos cerámicos en morteros romanos. Tradicionalmente, estos anillos se han vinculado con el potencial hidráulico de los morteros, pero sin cuantificar la alteración química que se produce en la interfaz del fragmento cerámico con la matriz de cal. Además de conocer el aumento de calcio en los áridos cerámicos, empleando variables diferenciales sobre una base geométrico-estadística, se 
caracterizaron los áridos desarrollando un mapping químico en la interfaz y una microscopía óptica polarizada en los áridos reactivos. Asimismo, se caracterizaron macroscópicamente los componentes de los morteros, tanto los áridos silíceos como los fragmentos de calcita triturada. Para ello se emplearon técnicas de aplicación de diferentes focos de incisión de luces oblicuas sobre las superficies, de tal forma que los cristales de cuarzo y de calcita reflejaban sombras distintas en función de las fracturas, lo que permitía diferenciar unos y otros granos con gtrandes amplificaciones, tal y como también desarrolla Luca Bertolini (Bertolini et al., 2013:11):

Calcite $(C)$ and Quartz $(Q)$ were detected in all the samples, and their peaks were always those of greater amplitude.

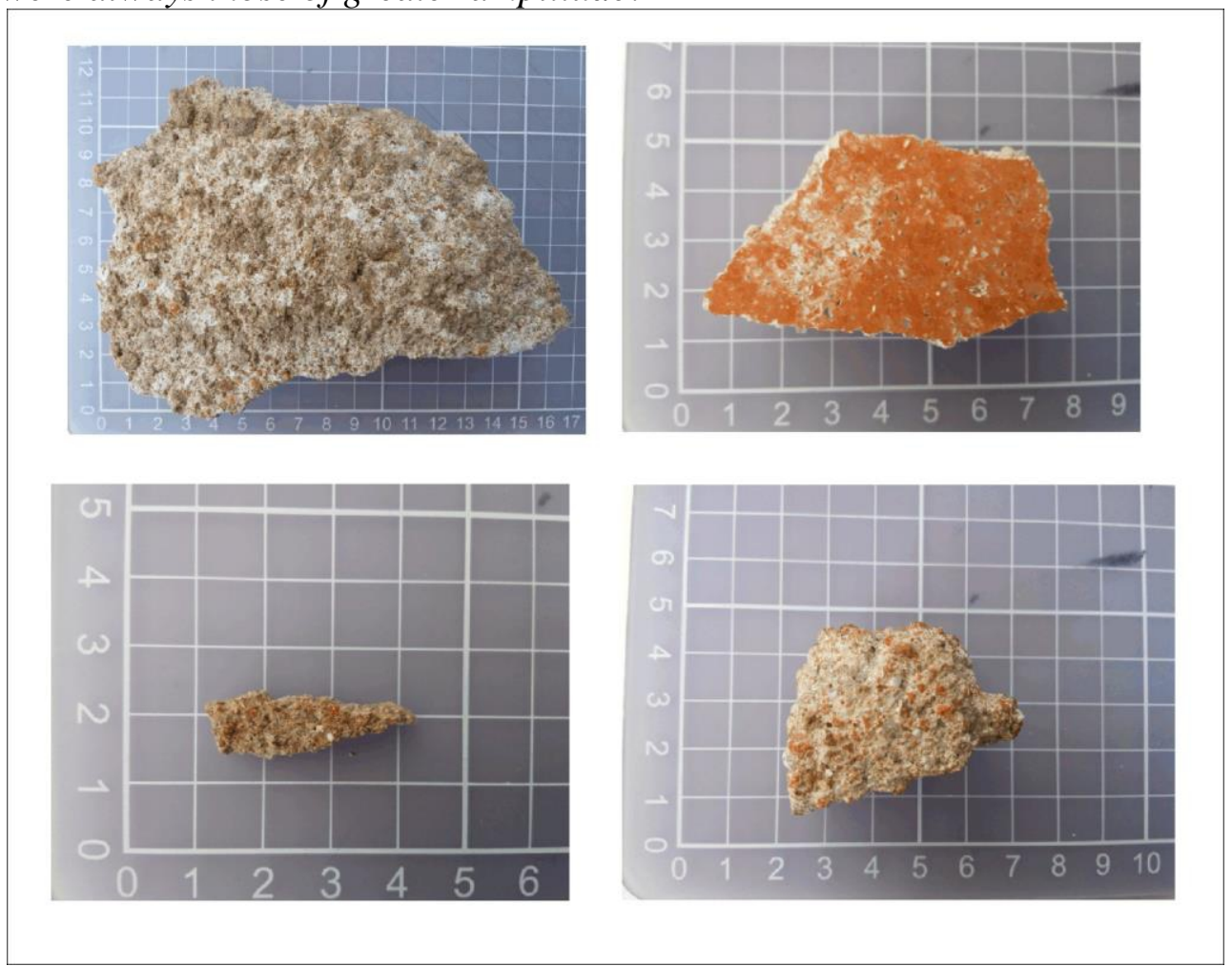

Fig. 3. Detalle de las muestras de mortero analizadas.

Este procedimiento, basado en el aspecto visual de la fractura, no es conclusivo y necesita de un análisis químico, tal y como indican algunos autores como Böke o Isikdag o Velosa, que insisten en la necesidad de aplicar análisis XRF o TD (Böke et al., 2006: 1118; Isikdag y Topçu, 2013; Velosa et al., 2007: 1215), o como concluye Bertolini, pues esos fragmentos podrían pertenecer a los áridos cerámicos, y no a los áridos silíceos (Bertolini et al., 2013: 12): 
Peaks of calcite and quartz are still evident. The latter, in this case, can be attributed to the presence of minute fragments of bricks.

También se desarrollaron en esta investigación diferentes esquemas de vectorización de los componentes, contando con algunos precedentes consultados (Balbas, 2002; Bertolini et al., 2013; Ricciardi et al., 2007).

Toda esta secuencia analítica queda integrada en la llamada arqueometría, en la que no vamos a profundizar, pero que cuenta con un bagaje bibliográfico tan extenso como variado en cuanto a los materiales arqueológicos analizados (Boschetti et al., 2008; Çizer, 2004). Las argamasas históricas, y en concreto, los morteros, cuentan con diversas investigaciones publicadas en las que se han aplicado los principios de la arqueometría, analizando entre otras, las características mecánicas o la micro-estratigrafía (Miriello, 2010; Guerra García y Sanz Arauz, 2013).

Para la identificación correcta de los componentes se acudió a la publicación de Mackenzie y Guilford, toda una monografía imprescindible para este campo (Mackenzie y Guilford, 1992). En cuanto a la identificación de los aditivos y los áridos, además de la bibliografía arriba mencionada, han sido consultados diversos manuales de microscopía de geomateriales (Ingham, 2013; Pecchioni et al., 2008). La lectura binocular se llevó a cabo con una Lupa Motic, modelo SMZ-140. Para las fases más pequeñas se empleó un microscopio USB Veho, modelo VMS-004 con aumentos 20x400 e iluminación de ocho LED's.

\section{Materiales y muestras}

Una vez analizadas las estructuras que fueron documentadas en el proceso de la excavación, se determinaron las muestras que debían ser procesadas, en función de los objetivos y las finalidades establecidos, es decir, diferenciar las distintas fábricas y manos de las opera, así como establecer un patrón relacionado con las unidades estratigráficas a las que pertenecen los morteros.

Por otro lado es interesante caracterizar los morteros con el objetivo global de crear modelos de trazabilidad, es decir, establecer una tipología general de las argamasas documentadas en yacimientos, de tal forma que se puedan hacer referencias cruzadas entre yacimientos, estructuras y cronologías, como ya se ha desarrollado en otros trabajos similares en yacimientos romanos (Prado et al., 2007), bizantinos (Meir et al., 2005), medievales (Ortega et al., 2009) e incluso mesoamericanos (Murakami et al., 2013). 


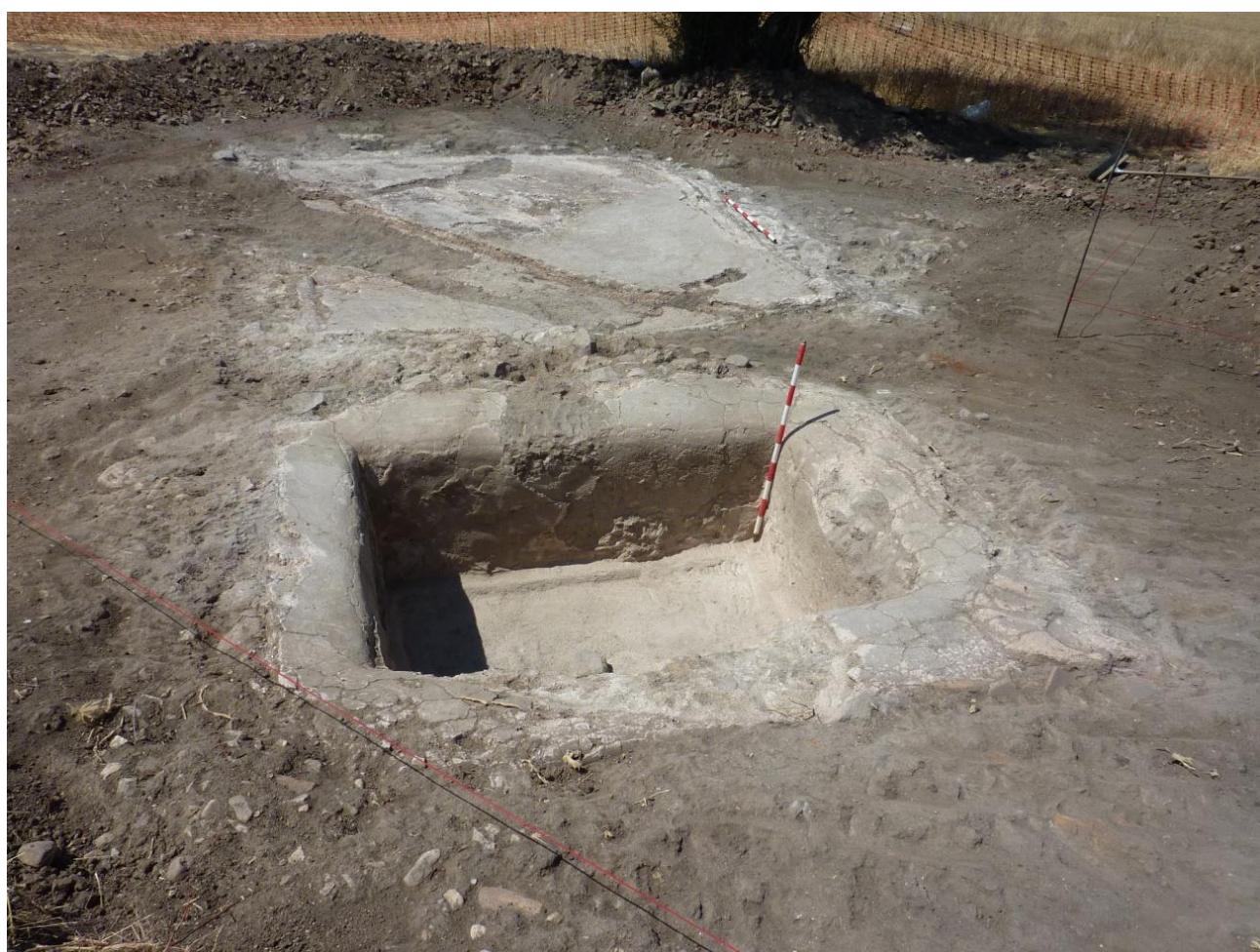

Fig. 4. Detalle de las estructuras del Sector 5, desde el sur, correspondientes con la UE 503 y UE 506.

Las muestras analizadas han sido las siguientes:

\begin{tabular}{|c|c|c|c|c|}
\hline Muestra & Tipo & Estructura & Punto & Apariencia \\
\hline VMT1 & Mortero de cal & $\begin{array}{c}\text { Estructura hidráulica 1 } \\
\text { UE 503 }\end{array}$ & $\begin{array}{c}\text { Pared } \\
\text { lateral }\end{array}$ & Opus signinum \\
\hline VMT2 & Mortero de cal & $\begin{array}{c}\text { Estructura hidráulica 1 } \\
\text { UE 504 }\end{array}$ & Relleno & $\begin{array}{c}\text { Opus Caementicium } \\
\text { enlucido }\end{array}$ \\
\hline VMT3 & Mortero de cal & $\begin{array}{c}\text { Estructura hidráulica 1 } \\
\text { UE 504 }\end{array}$ & Relleno & Opus signinum \\
\hline VMT4 & Mortero de cal & $\begin{array}{c}\text { Estructura hidráulica 2 } \\
\text { UE 506 }\end{array}$ & Relleno & Opus signinum \\
\hline
\end{tabular}

\section{Resultados}

\section{VMT1}

La muestra VMT1 corresponde a un mortero de cal con áridos silíceos y cerámicos en una distribución aproximada de 1:3, y de la clase opus signinum.

En el análisis de visu no se han localizado elementos destacables, aunque la muestra es consistente al tacto. Además, en apariencia, el fragmento podría corresponder a alguna moldura tipo "media caña", por la curvatura convexa de la placa. No se han observado pigmentaciones ni 
enlucidos ni residuos orgánicos que puedan ser susceptibles de ser aislados. Se procedió a la limpieza en seco y en húmedo.

El aglutinante es la cal, siendo ésta de color blanquecino-grisáceo, con un aspecto aparentemente limpio salvo por la presencia de algunos nódulos de tamaño considerable. Los áridos identificados son arenas de morfología silícea, propia de ambientes fluviales: cuarzos, feldespatos y micas en su mayoría. Los áridos cerámicos están formados por fragmentos de arcilla cocida a altas y bajas temperaturas, siendo fragmentos muy aristados y de tamaño pequeño (entre 100 y 500 micras). La textura es rugosa y las pastas toscas (desoxidantes de gran tamaño).

La ordenación de los granos es regular presudoisódroma, con un tamaño máximo en los áridos cerámicos. Las arenas tienen un tamaño menor y se distribuyen en una forma más o menos ordenada. Se aprecia cierta selección de los granos (escasamente aristados, granos redondeados, poca desviación en la media de tamaños, etc.). Según la clasificación Raymond estaríamos ante un árido semi-anguloso.

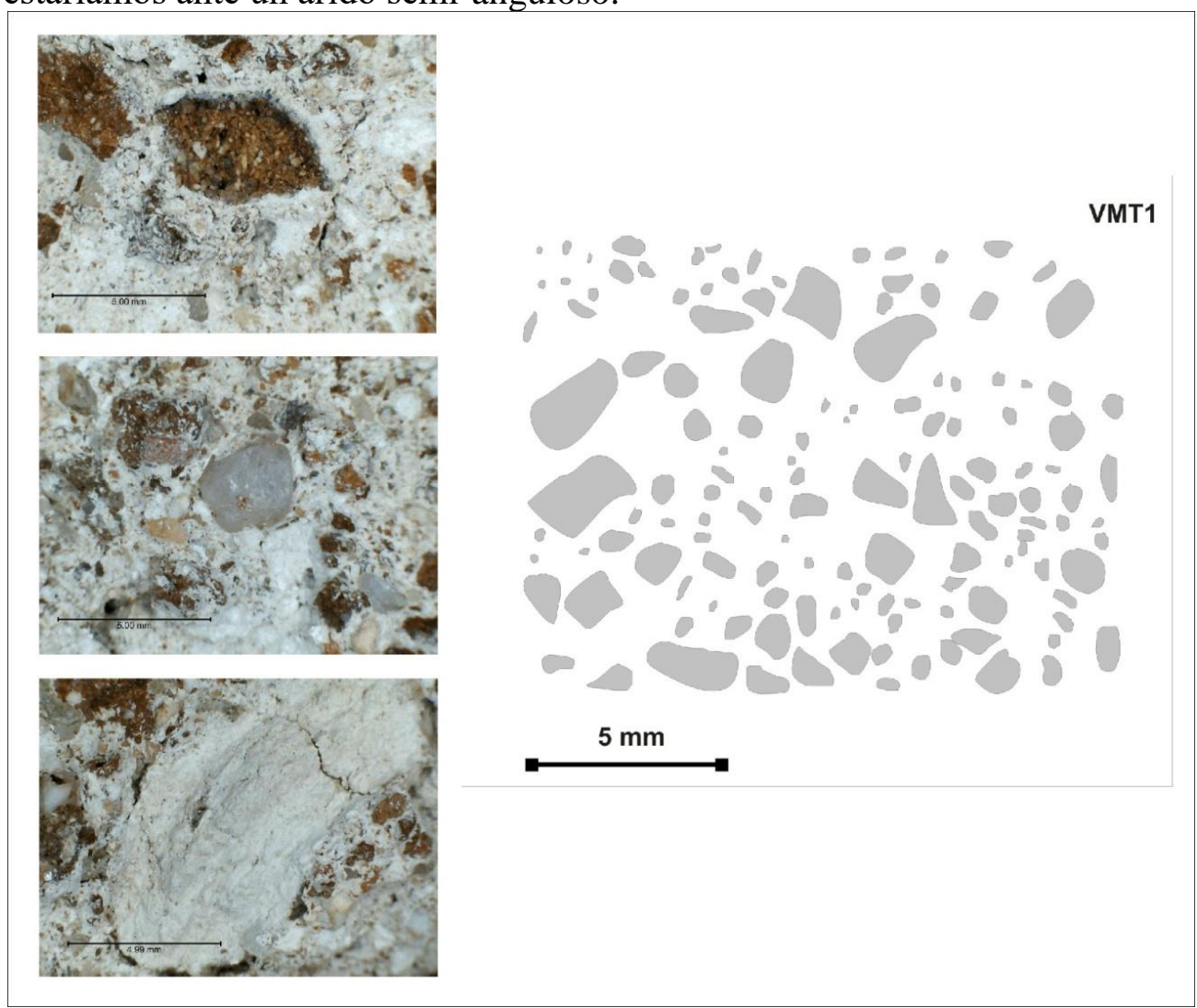

Fig. 5. Caracterización y granulometría media de la muestra VMT1.

Se han localizado algunas fibras vegetales en el interior de la matriz. Por el contrario no se han observado degradaciones ni intrusiones orgánicas 
destacables, salvo algunas oxidaciones en la superficie del exterior, más propia de la recarbonatación del aglutinante expuesto a la intemperie.

\section{VMT2}

La muestra VMT2 se ha identificado como un mortero de cal con áridos silíceos en una distribución aproximada de 1:3, con ausencia de áridos cerámicos, por lo que estaríamos ante un mortero del tipo opus caementicium. En el análisis de visu se ha identificado una fase de revestimiento pigmentado. La muestra es poco consistente al tacto en el lado interior de la fábrica. La pieza podría corresponder con un mortero enlucido al fresco sobre un capeado de mortero de áridos muy finos. Tras observar ese elemento se procede a la limpieza en seco sin aplicar ningún producto químico.

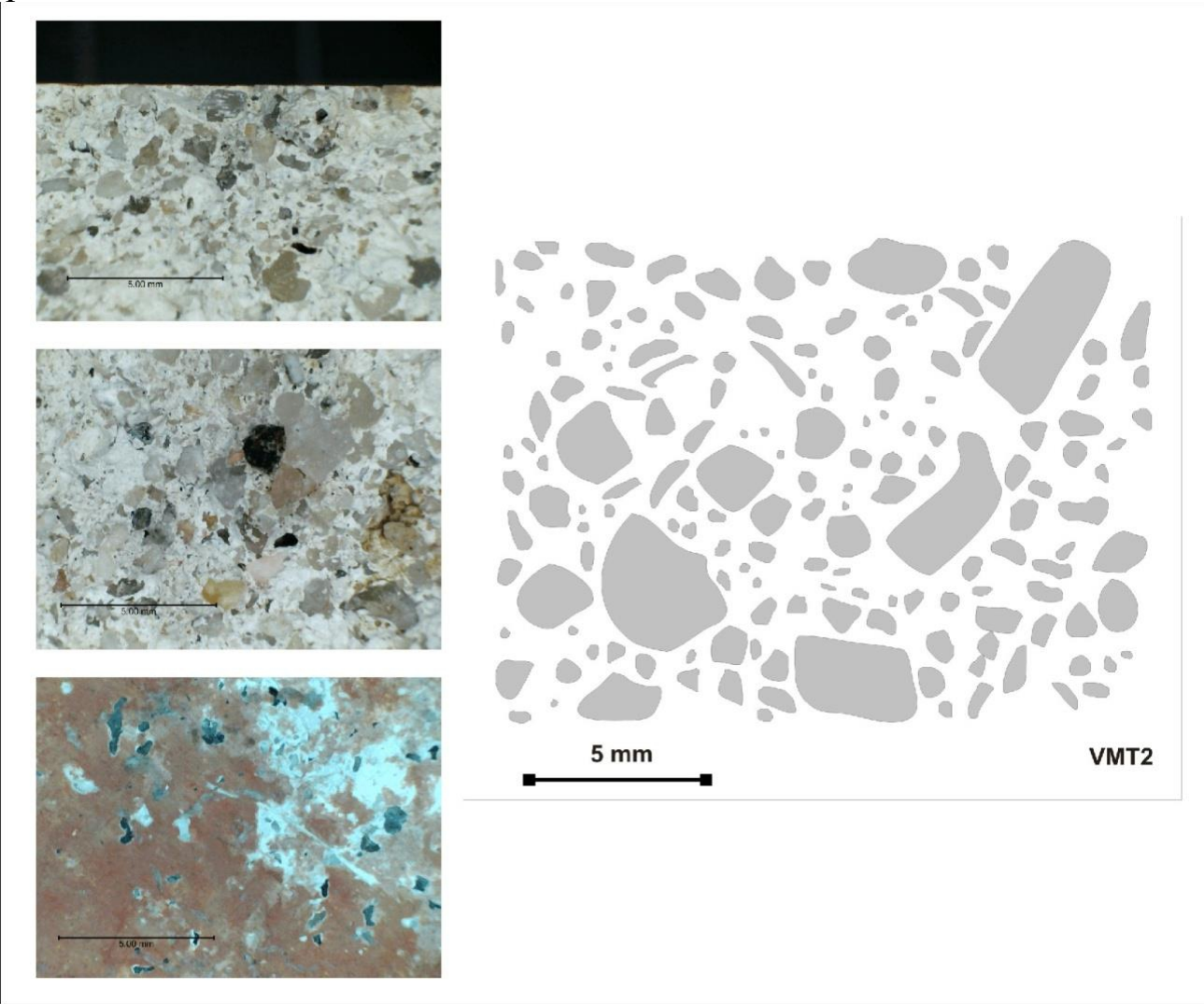

Fig. 6. Detalle de las características de la muestra VMT2.

Como aglutinante se utiliza la cal, de un color blanquecino y con un aspecto aparentemente limpio, sin apenas presencia de nodulaciones salvo en la parte interna. Los áridos también son arenas de morfología silícea, localizadas en ambientes fluviales, principalmente con cuarzos, feldespatos y micas en su mayoría, identificando alguna mica biotita. 
En cuanto a la ordenación de los componentes, los granos se distribuyen de forma regular y presudoisódroma, aunque con tamaños más aproximados a la media que en la muestra VMT1. Las arenas tienen un tamaño pequeño/mediano (entre 150 y 700 micras aproximadamente) con una distribución bastante ordenada. Al igual que en la anterior muestra, se observa una selección de los granos más aparente (pocas aristas, granos redondeados, escasa desviación en la media de tamaños, etc.).

En lo referido a las capas o fases constructivas, la UC1 (exterior) apenas llega a tener un espesor visible a la lupa binocular, por lo que debiera ser inferior a las 100 micras. Del resto de las fases, es decir, UC2 (cama) desconocemos el espesor total.

\section{VMT3}

La muestra VMT3 ha sido identificada como un mortero de cal con áridos silíceos y también cerámicos en una distribución aproximada de 1:3, por lo que sería una argamasa del tipo opus signinum. Tras inspeccionar la muestra de visu no se han identificado fases constructivas, pero sí que es muy poco consistente al tacto, presentando grandes cantidades de tierra adherida a la misma y perdiendo parte del material, principalmente áridos. Este tipo de mortero ha sido difícil de caracterizar, principalmente por su conservación.

Ante la presencia de áridos cerámicos debería corresponder con un hipotético mortero con cierta capacidad hidráulica, pero sorprende la poca resistencia al agua, ya que tras la limpieza en seco y en húmedo, en la segunda fase la muestra se reduce al $70 \%$ por degradación de los materiales arcillosos, por lo que se preserva la cantidad necesaria para realizar la macroscopía sin riesgo de degradación completa.

El aglutinante empleado es la cal, y se presenta con un color blanquecino oscuro/marrón y con un aspecto poco limpio. Se han apreciado muchas concreciones calcáreas y al tacto es usual la pérdida de material (del árido, preferentemente).

Los áridos utilizados son arenas silíceas de ambientes fluviales, principalmente con cuarzos, feldespatos y micas en su mayoría, mientras que los áridos cerámicos, de pequeño tamaño $(<1 \mathrm{~mm})$ corresponden a arcillas escasamente cocidas. Se aprecian pocas aristas en los fragmentos cerámicos, al igual que en los áridos silíceos, lo que responde a un tipo semi-circular según la clasificación Raymond.

En lo referido a la ordenación de los componentes, los granos se distribuyen de forma aparentemente regular y presudoisódroma, muy similar a la muestra VMT1 pero con tamaños más reducidos en las arenas (entre 200 y 1000 micras aproximadamente) y con una distribución ordenada. Al igual 
que en las muestras anteriores también se observa una selección de los granos, incluso en los áridos cerámicos (en general, poco aristados).

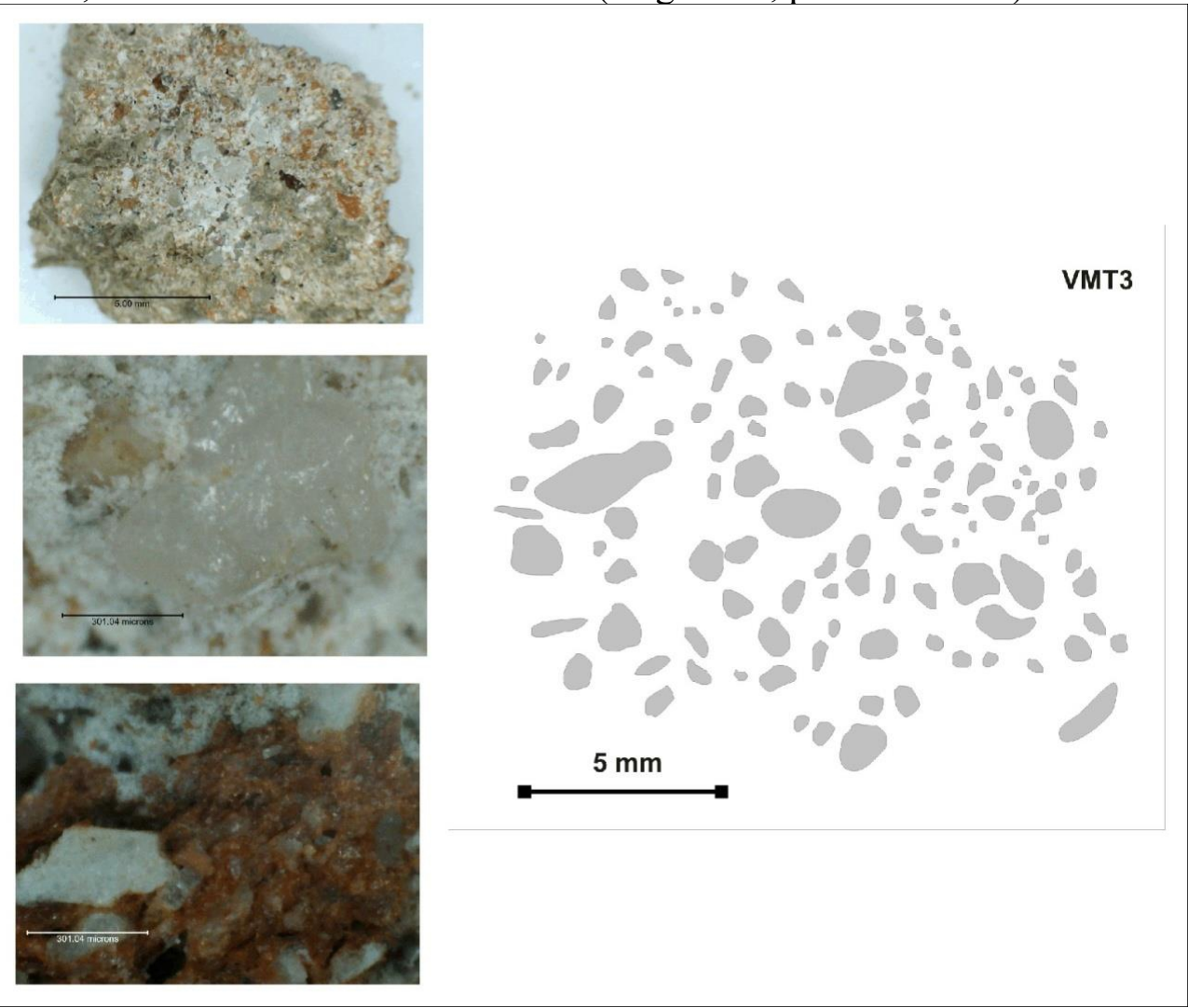

Fig. 7. Aspecto de la muestra VMT3, caracterizada y con la granulometría completa.

\section{VMT4}

Finalmente la muestra VMT4 se ha identificado como un mortero de cal con áridos silíceos e igualmente con la presencia de áridos cerámicos, en una distribución aproximada de 1:3, por lo que nuevamente sería de la clase opus signinum. Con el análisis de visu se ha apreciado que la muestra es consistente al tacto, aunque se desprenden algunos fragmentos de cerámica, poco adheridos al aglutinante. A pesar de presentar un aspecto tosco podría corresponder a un hipotético mortero hidráulico. 

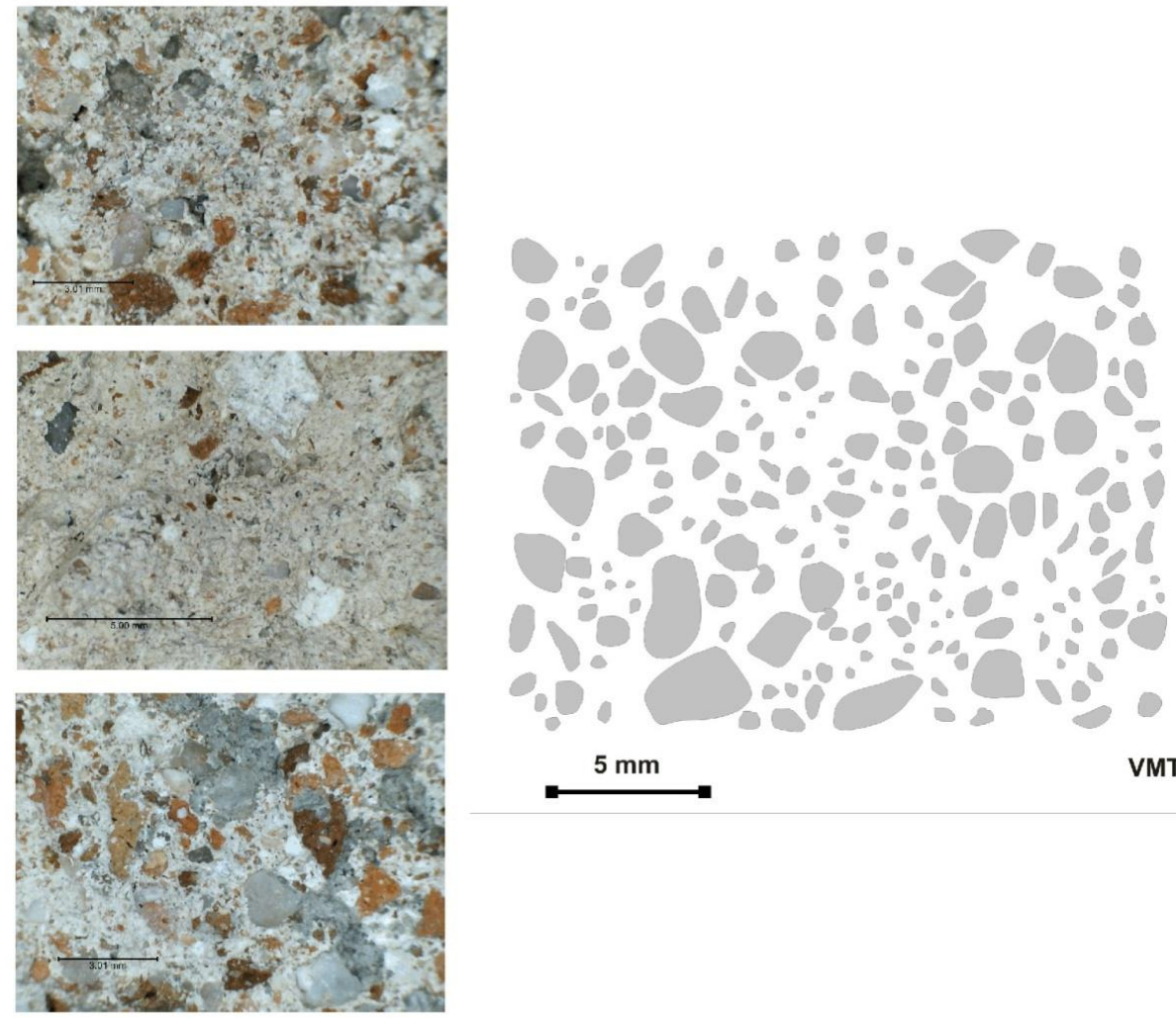

VMT4

Fig. 8. Muestra VMT4, con la granulometría y sus principales componentes visualizados.

El aglutinante utilizado, al igual que en las anteriores muestras, es la cal, y presenta un color blanquecino/grisáceo con un aspecto aparentemente limpio. No se han observado grandes nodulaciones calcáreas ni intrusiones, aunque durante los ensayos se desprendieron algunos áridos.

También los áridos son de la misma naturaleza mineralógica que en las muestras anteriores, es decir, arenas de morfología silícea de ambientes fluviales, con cuarzos, feldespatos y micas. En lo referido a los áridos cerámicos son de pequeño tamaño, salvo alguna excepción (entre 300 micras y $3 \mathrm{~mm}$ ). Todos corresponden a arcillas poco cocidas y de forma irregular. Las pastas son de poca calidad, con desoxidantes de mediano tamaño.

Por su parte, la ordenación de los componentes es ligeramente regular y presudoisódroma, con tamaños pequeños en las arenas (entre 200 y 1000 micras aproximadamente). Los granos se distribuyen de forma ordenada, tanto las arenas como las arcillas cocidas. Como sucede en las demás muestras también se observa cierta selección de los granos (poco aristados). En cuanto a la clasificación de los áridos se enclavarían en los semicirculares según la clasificación Raymond. 


\section{Interpretaciones}

Una vez analizados los morteros, y sobre la base de los resultados obtenidos, si bien es cierto que los análisis se centran en la observación macroscópica y en la caracterización física de los componentes, las concusiones obtenidas enriquecen los datos arqueológicos obtenidos de la intervención, sin los cuales, la caracterización de morteros no tendría sentido.

En primer lugar, y en función de los componentes básicos que se han localizado, se podría decir lo siguiente:

- Áridos: Son por lo general arenas de origen fluvial; áridos seleccionados de cuarzos, feldespatos y micas. Los áridos de la muestra VMT2 presenta un aspecto más limpio y un tamaño más pequeño (mayor cribado en la selección). Las muestras VMT1, VMT3 y VMT4 tienen características hidráulicas, ya que se han encontrado áridos cerámicos en la mezcla. La hidraulicidad de los morteros acarrea muchas investigaciones. Esta característica se conseguía, o bien empleando una cal que fuese hidráulica per se, o incluyendo compuestos que potenciasen la hidraulicidad, tales como fragmentos cerámicos o puzzolana. En España es mucho más frecuente la cerámica o también llamada "cocciopesto". A grandes rasgos, la reacción entre la cerámica y la cal produce un carbonato sílice aluminato. Si un mortero es hidráulico se debe claramente a una intencionalidad, y por lo tanto se busca una reacción positiva frente al agua.

- Aglomerante: Todas las argamasas cuentan, como elemento aglomerante principal, la cal. De las cales, la documentada en la muestra VMT3 está peor decantada que las restantes, a la vista del color y la firmeza con respeto a los áridos (pérdida de piezas). Del conjunto, la que tiene mejor aspecto (mezcla, color, distribución, menor presencia de nódulos...) es la VMT2, probablemente porque corresponde con una lechada exterior como cama a un enlucido. No se sabe cuál es la principal fuente de extracción de cales de la zona, pero lo normal es emplear como conglomerante el recurso natural más abundante.

- Otros componentes: Las muestras analizadas no presentan otros áridos como gravas, áridos basálticos o aditivos, salvo de forma aislada algunos componentes orgánicos como fibras vegetales (VMT1), probablemente incluidos en la masa sin intencionalidad alguna (tamaño muy reducido).

- No se han apreciado bioataques ni intrusiones orgánicas entre los áridos. Tan solo mencionar alguna reacción química más propia de la oxidación de los cuarzos. 


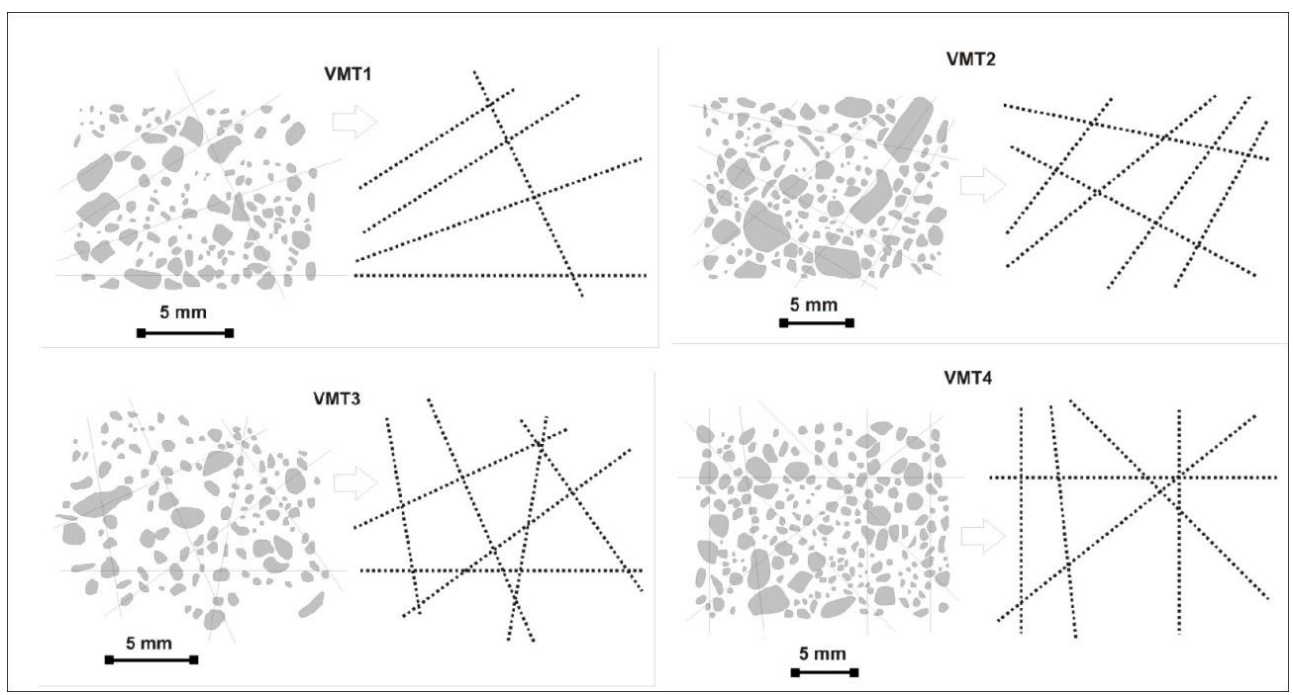

Fig. 9. Tipología de las granulometrías obtenidas de las muestras de mortero. siguientes:

En cuanto a las granulometrías, las interpretaciones podrían ser las

- $\quad$ Las muestras VMT1, VMT2 y VMT4 presentan una granulometría más ordenada y con unos vectores de distribución de áridos más regulares, según las pautas de Ingham. Este factor no coincide con el tamaño medio de los granos, ya que la muestra VMT1 tiende a un aumento del tamaño medio de los áridos, con una distribución quizá más desigual que en el resto de las muestras.

- $\quad$ Por su parte la muestra VMT2, correspondiente con un mortero de cama para un revestimiento, presenta una distribución muy regular, con escasa acumulación de áridos y un alineamiento de los mismos muy ordenada.

- Las muestras VMT1 y VMT4, ambos del tipo opus signinum, presentan unas retículas muy similares, y por lo tanto, una "mano" parecida en la fábrica, a excepción del tamaño medio de sus áridos.

Finalmente, en cuanto a la microestratigrafía se refiere, los resultados se podrían resumir de la siguiente manera:

- Solo una argamasa ha documentado una microestratigrafía clara, la VMT1. Presenta una capa exterior de revestimiento con pigmentación, y se caracteriza por una oxidación en los niveles al contacto con la atmósfera, algo normal teniendo en cuenta los factores de re-carbonatación que sufren las cales.

- $\quad$ El espesor de la capa UC1 es mínimo, y sólo podría ser cuantificable a escala microscópica.

- Las características mineralógico-químicas del pigmento solo podrían ser cuantificables por medio de un análisis de espectro. Se puede decir que la 
coloración es rojiza y que se aprecian marcas de microespatulado en el exterior. No se aprecian figuras ni composiciones. Visualmente podría ser una pintura acuosa aplicada sobre una lechada de mortero muy rico en cal y escaso en áridos.

En resumen, se podrían sacar varias líneas conclusivas. Por ejemplo, en lo que respecta a los deterioros visibles en las muestras de mortero, los análisis de visu han determinado que el ligado de los áridos es bueno en términos generales, salvo en la muestra VMT3, con un considerable desprendimiento de material al tacto. En este último caso supone que la calidad del aglomerante, unido a los factores externos de deterioro, no es buena, y ello puede deberse o bien a una mala cochura de las calizas y por lo tanto, una mala calidad de la cal, o bien por una incompatibilidad de la cal, una vez fraguado el mortero, con las condiciones climatológicas, la acidez del suelo, etc. Hay diversos precedentes al respecto y se conocen tanto las causas como las consecuencias de este desgaste (Magalhaes y Veiga, 2009; Sabbioni et al., 2002), así como los procedimiento necesarios para su restauración (Arioglu y Acun, 2006).

En lo que respecta a los áridos, es obvio el empleo de arenas de procedencia fluvial, bien de granulometría pequeña o grande, como viene sucediendo en la mayoría de los morteros de cronología romana. La selección de los áridos silíceos, e incluso de otros áridos como los puzolánicos, condiciona las características y usos que se le atribuye a cualquier tipo de argamasa, como por ejemplo explica Antonia Moropoulou (Moropoulou et al., 2003: 892):

This technology [crushed brick limes] spans from early Byzantine to Venetian and late Ottoman periods and gives mortars with excellent pozzolanic properties due to the adhesion reactions at the ceramic-matrix interface. "Cementitious" mortars represent "opus caementicium' of Vitruvius, which was described as an artificial conglomerate of gravel with sand, lime and pozzolana (volcanic earth).

En lo relativo a los morteros de Villamanta, las dosificaciones de los áridos mantienen los estándares más comunes. Cabe destacar la diferencia de los áridos del mortero con pigmentación (VMT2), con una granulometría más perfecta, simétrica y ordenada. 


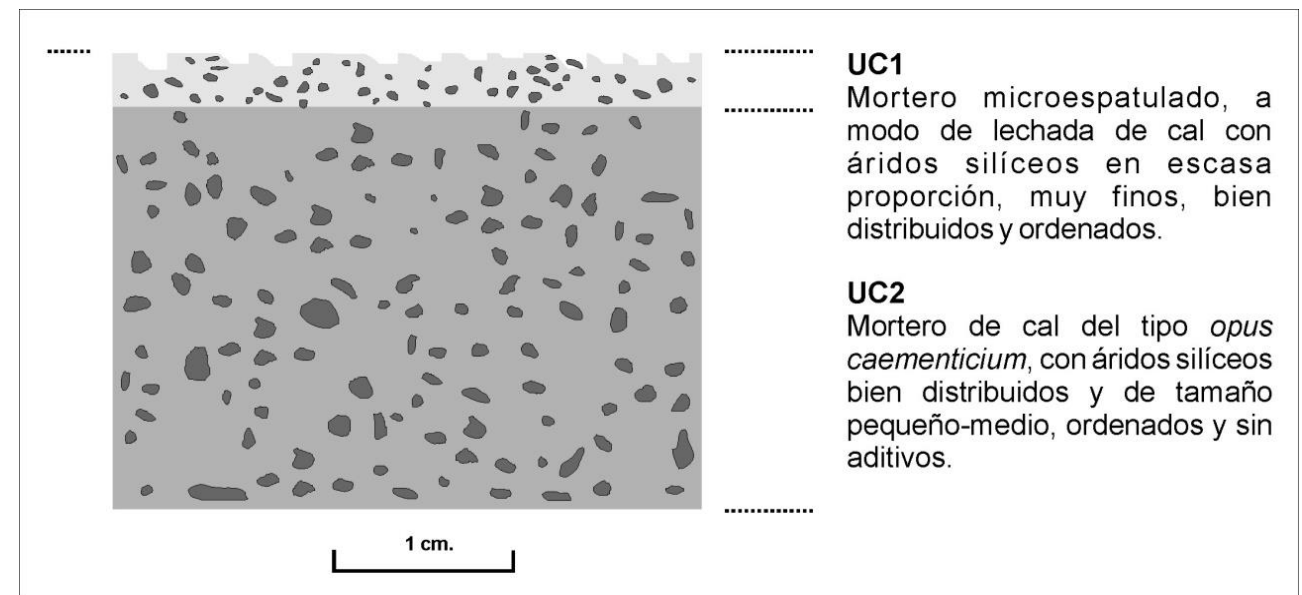

Fig. 10. Microestratigrafía desarrollada en la muestra VMT2, en donde se ha documentado una capa pictórica exterior.

La presencia de áridos cerámicos, con una granulometría similar en todos los casos, prevé un uso industrial en los morteros. Algunos investigadores insisten en la importancia de la homogeneidad de las calidades y dosificaciones, el verdadero éxito de los morteros de época romana (Álvarez Galindo et al., 1995: 55). La presencia de áridos cerámicos queda atestiguada en innumerables yacimientos, si bien es cierto que las investigaciones han demostrado su importancia en el comportamiento hidráulico en morteros industriales (Bugini, 1991; González Cortina, 2000; Zendri et al., 2004; Guerra García, 2015).

\section{Conclusion}

La caracterización física y macroscópica de los morteros forma parte de la primera fase de un largo proceso analítico y deductivo, tal y como determinan la mayoría de los estudios consultados (Alejandre Sánchez et al., 2004; Miriello, 2006). Caracterizar un mortero significa identificar sus componentes y comprender cómo interactúan éstos. El estudio de caracterización que aquí se ha llevado a cabo ha permitido obtener unos resultados muy valiosos desde el punto de vista global del yacimiento, ya que las cronologías dadas en la investigación arqueológica pueden ser cruzadas con las características propias del mortero, generando, por un lado, una tabla tipológica de morteros por cronología, y por otro lado establecer patrones de fabricación por tipos de estructuras.

Asimismo se han documentado algunos elementos que determinan el estado de conservación de los morteros, en tanto que dicho estado de conservación queda condicionado por la calidad de las manufacturas, como la presencia de poro o de nódulos, que condiciona sus características físicas y mecánicas (Arandigoyen, y Álvarez, 2007), o el tamaño y dosificaciones de 
los áridos, que pueden determinar la respuesta de los morteros ante las tensiones (Hormes et al., 2016: 191).

Salvo la muestra VMT3, todas las muestras son consistentes, y todas corresponden a morteros de carácter industrial -opus signinum- a excepción de la VMT2, que se refiere a un mortero del tipo opus caementicium.

Todas las muestras emplean un árido silíceo de procedencia fluvial, y fragmentos de cerámica en las del tipo signinum, con pastas arcillosas muy irregulares y con desoxidantes de tamaño medio. Ello podría suponer que proceden del machaqueo de material constructivo como tejas o ladrillo. No se han identificado otros áridos ni aditivos, salgo algunas fibras vegetales.

La muestra VMT2 presenta un revestimiento con pigmentación de color rojiza-anaranjada. El espesor de la capa exterior es mínimo -poca penetración en la cama del mortero-. En esta muestra la cal es, en apariencia, de buena calidad.

En lo referido al aglomerante, todos son cales de calidad buena, bien decantadas y con presencia puntual de nódulos de mediano tamaño, más numerosos en las muestras VMT1 y VMT4.

\section{References:}

1. Alejandre Sánchez, F. J.; Enríquez Díaz, C.; Martín Del Río, J. J. y Flores Alés, V. (2004): Restauración de un mosaico romano: caracterización y análisis constructivo. En PH: Boletín del Instituto Andaluz del Patrimonio Histórico, año XII, 51 (pp. 94-101). Sevilla: Instituto Andaluz de Patrimonio Histórico.

2. Alvar Ezquerra, A. (Coord. 1993). Relaciones topográficas de Felipe II. Madrid: Comunidad de Madrid y C.S.I.C.

3. Álvarez Galindo, J.I.; Martín Pérez, A. y García Casado, P.J. (1995): Historia de los morteros. En Boletín Informativo del Instituto Andaluz de Patrimonio Histórico, 13 (pp. 52-59). Sevilla: Junta de Andalucía.

4. Arandigoyen, M. y Álvarez, J.I. (2007): Pore structure and mechanical properties of cement-lime mortars. En Cement and Concrete Research, 37 (pp. 767-775). Elsevier.

5. Ariuglu, N, y Acun, S. (2006): A research about a method for restoration of traditional lime mortars and plasters: A staging system approach. En Building and Environment, 41 (pp. 1223-1230). Elsevier.

6. Arkatros (2011). Proyecto de abastecimiento de urgencia en Villamanta (TT.MM.:Navalcarnero y Villamanta). Memoria final de prospección arqueológica. Madrid. Inédito.

7. Bartos, P., Groot, C. Y Hughes, J. J. (eds. 2000): Historic mortarscharacteristics and test. En Proceeding of the International RILEM Workshop, Escocia, 1999. Cachan: RILEM. 
8. Bermúdez, A. y Marqués, I (2016). Poblamiento romano en la vega del Arroyo Grande: El Tejar (Villamanta). En RAM 2016. Reunión de Arqueología madrileña (Alcalá de Henares, 23-25 de noviembre de 2016). Actas en prensa.

9. Bertolini, L.; Carsana, M.; Gastaldi, M.; Lollini, F. y Redaelli, E. (2013): Binders characterization of mortars used at different ages in the San Lorenzo church in Milan. En Materials Characterization, 80 (pp. 9-20). SciencDirect.

10. Böke, H.; Akkurt, S.; Ipekoglu, B. y Ugurlu, E. (2006): Characteristics of brick used as aggregate in historic brick-lime mortars and plasters. En Cement and Concrete Research, 36 (pp. 1115-1122). Elsevier.

11. Boschetti, C., Leonelli, C., Macchiarola, M., Veronesi, P., Corradi, A. y Sada, C. (2008): Early evidences of vitreous materials in Roman mosaics from Italy: An archaeological and archaeometric integrated study. En Journal of Cultural Heritage, 9 (pp. 21-26). EM Consulte.

12. Broekmans Maarten, A. T. M. (2009): Petrography as an essential complementary method in forensic assessment of concrete deterioration: two case studies. En Materials Characterization, 60 (pp. 644-654). Elsevier.

13. Bugini, S. (1991): Investigation of the characteristics and properties of Cocciopesto from the ancient Roman period. En Conservation of stone and other materials, 1 (pp. 386-93). Thiel: RILEM proceedings.

14. Carta Arqueológica de la Comunidad de Madrid. Madrid: Dirección General de Patrimonio Histórico. Comunidad de Madrid.

15. Contreras, M. (1993). Aproximación al estudio de la Mantua Carpetanorum de Ptolomeo: nuevas aportaciones arqueológicas para su localización. En XXII CNA, 2 (pp. 399-404). Vigo: Xunta de Galicia.

16. Çizer, O. (2004): Investigation of Lime Mortar Characteristics for the Conservation of the Ottoman Baths in Seferihisar-Urla Region. En: Dissertation Submitted to the Graduate School in Partial Fulfillment of the Requirements for the Degree of Master Of Science. İmir: İzmir Institute of Technology.

17. Doebley, C. E. y Spitzer, D. S. (1996): Guidelines and standards for testing historic mortars. En Kelly, S. J. (coord.): Standards for preservation and rehabilitation, (pp. 285-293). Filadelfia: ASTM STP, American Society for Testing Materials.

18. Elsen, J. (2005): Microscopy of historic mortars: a review. En Cement and Concrete Research, Actas del X Euroseminar on 
Microscopy Applied to Building Materials, June 21-25, 2005, volumen 36, capítulo 8 (pp. 1416-1424). University of Paisley.

19. Fernández, F.J. (2007). Informe de actuación arqueológica, calle San Blas, 16. Inédito.

20. Fernández, F.J. (2008). Informe de actuación arqueológica, calle San Blas, 16. Villamanta. Inédito.

21. Fernández, F.J. (2010). Informe de actuación arqueológica, calle Real, 5 de Villamanta Inédito.

22. Gamallo, J.L. y Gimeno, H. (1990). Nuevas inscripciones romanas de Villamanta (Madrid). En Gerión, 8, (pp. 287-298). Madrid: Universidad Complutense de Madrid.

23. Gómez-Reino, C. et al. (1994). Intervención arqueológica en Villamanta (Madrid). En Boletín del Ilustre Colegio Oficial de Doctores y Licenciados en Filosofía y Letras y en Ciencias, 55, XIXII. Madrid: Colegio de Doctores y Licenciados en Filosofía y Letras y en Ciencias de Madrid.

24. Guerra García, P. y Sanz Arauz, D. (2013): Metodología arqueológica aplicada al estudio de los materiales constructivos históricos. El ejemplo de los morteros romanos en España. En Actas del I Congreso REUSO, International Congress on Conservation, Restoration and Reuse of Architectural Heritage, volumen I (pp.423431). Madrid: Servicio de Publicaciones. Università degli Studi di Firenze - UPM.

25. Guerra García, P. (2015): Sola Romani. Los morteros hidráulicos romanos en la península Ibérica. Tesis Doctoral Inédita. Madrid: ETSAM. Universidad Politécnica de Madrid.

26. Gülec, A. y Tulun, T. (1997): Physico-chemical and petrographical studies of old mortars and plasters of Anatolia. En Cement and Concrete Research, A, 27, 2 (pp. 227-234). Elsevier.

27. Herrera, T. y Velasco, J.F. (2003). Informe preliminar de actuación y dictamen sobre la excavación arqueológica realizada en el proyecto de acondicionamiento de la carretera M-507. Tramo VillamantaAldea del Fresno. Inédito.

28. Hoces de la Guardia, A.L.; Bermúdez, A. y Marqués, I. (2015). Intervención arqueológica en "El Tejar" (Villamanta, Madrid): las piezas epigráficas de época romana. En Vides Monumenta Veterum. Alcalá de Henares. Actas en prensa.

29. Hughes J. y Callebaut, K. (2000): Practical sampling of historical mortars. En Bartos, P.; Groot, C. y Hughes, J.J. (eds.): Proceedings of the RILEM International Workshop Historic Mortars: characteristics and tests, (pp. 17-26). university of Paisley. 
30. Ingham, J. (2010): Geomaterials under the microscope. A colour guide. Londres: CRC Press.

31. Isikdag, B y Topçu, I. B. (2013): The effect of ground granulated blast-furnace slag on properties of Horasan mortar. En Construction and Building Materials, 40 (448-453). Elsevier.

32. Jedrzejewska, H. (1960): Old mortars in Poland: a new method of investigation. En Studies in Conservation, 5 (pp. 132-138). Hampshire: Taylor \& Francis Group.

33. López, T. (1788). Geografia histórica de España. Provincia de Madrid, II. Madrid.

34. Magalhaes, A y Veiga, R. (2009): Caracterización física y mecánica de los morteros antiguos. Aplicación a la evaluación del estado de conservación. En Materiales de Construcción, volumen 59, 295 (pp. 61-77). Madrid: Instituto de Ciencias de la Construcción Eduardo Torroja. CSIC.

35. Mackenzie, W. S. y Guilford, C. (Ed. 1992): Atlas de Petrografía. Madrid: Ed. Marceliano Lago San José y Enrique Arranz Yagüe. Masson S.A.

36. Marqués, I.; Bermúdez, A. y Hoces de la Guardia, A.L. (2016). Villamanta y el yacimiento de "El Tejar". Primeras aportaciones. En Actas RAM 2014. Reunión de Arqueología Madrileña (Alcalá de Henares, 20-21 de noviembre de 2014) (pp. 203-211). Madrid: Colegio de Doctores y Licenciados en Filosofía y Letras y en Ciencias de la Comunidad de Madrid y Dirección General de Patrimonio Histórico.

37. Martínez, V. (2006). Memoria de la actuación arqueológica en la parcela 14 de la urbanización de La Mampuesta (Villamanta. Madrid). Inédita.

38. Meir, I. A.; Freidin, C. y Gilead, I. (2005): Analysis of Byzantine mortars from the Negev Desert, Israel, and subsequent environmental and economic implications. En Journal of Archaeological Science A, 32, 5 (pp. 767-773). Elsevier.

39. Mertens, G., Elsen, J., Brulet, R., Brutsaert, A. y Deckers, M. (2009): Quantitative composition of ancient mortars from the Notre Dame Cathedral in Tournai (Belgium). En Materials Characterization, 60 (pp. 580-585). Elsevier.

40. Miriello, D. (2006): Image analysis and flatbed scanners. A visual procedure in order to study the macro-porosity of the archaeological and historical mortars. En Journal of Cultural Heritage, 7 (pp. 186192). Elsevier.

41. Miriello, D. (2010): Characterisation of archaeological mortars from Pompeii (Campania, Italy) and identification of construction phases 
by compositional data analysis. En Journal of Archaeological Science, 37, 9 (pp. 2207-2223). Elsevier.

42. Miriello, D.; Bloise, A.; Crisci Gino, M.; Apollaro, C.; La Marca, A. (2011): Characterization of archaeological mortars and plasters from Kyme (Turkey). En Journal of Archaeological Science, 38, 4 (pp. 794-804). Elsevier.

43. Montero, J. (1990). La Carpetania en Ptolomeo. En Toledo y Carpetania en la Edad Antigua. Toledo.

44. Moropoulou, A.; Polikreti, K.; Bakolas, A. y Michailidis, P. (2003): Correlation of physicochemical and mechanical properties of historical mortars and classification by multivariate statistics. En Cement and Concrete Research, 33 (pp. 891-898). Elsevier.

45. Murakami, T.; Hodgins, G. y Simon, A.W. (2013): Characterization of lime carbonates in plasters from Teotihuacan, Mexico: preliminary results of cathodoluminescence and carbon isotope analyses. En Journal of Archaeological Science, 40 (pp. 960-970). Elsevier.

46. Ortega, L.A.; Zuluaga, M.C.; Insausti, M.; Alonso, A. e Ibáñez, A. (2009): Morteros arqueológicos de Santa María la Real (Zarautz, Guipúzcoa): evidencias de diferentes procedencias de la cal. En MUNIBE, 28 (pp. 162-177). San Sebastián: SC Aranzadi.

47. Oñate, P. (2001a). Informe final. Intervención arqueológica. Plan Parcial S.A.U. 5. Villamanta. Inédito.

48. Oñate, P. (2001b). Informe preliminar de la excavación arqueológica (2 ${ }^{a}$ fase). Plan Parcial S.A.U. 5. Villamanta. Inédito.

49. Pecchioni, E.; Fratini, F. y Cantisani, E. (2008): Le malte antiche e moderne: tra tradizione ed innovazione. Bolonia: Pàtron Editore.

50. Pellicer, J.A. (1803). Disertación histórico-geográfica sobre el origen y población de Madrid: así en tiempos de moros como de cristianos. Madrid.

51. Prado Govea, R. H.; Louis, M.; Huesca, J. A.; Uróz, J.; García del Cura, M. A. y Spairani, Y. (2007): Caracterización y estudio, de los morteros y materiales pétreos, en las ruinas de la ciudad de Pompeya. En IX Congreso Latinoamericano de Patología XI Congreso de Calidad en la Construcción (pp. 1-8). Quito: ALCONPAT.

52. Ptolomeo. Geographia. En Hispania antigua según Pomponio Mela, Plinio el Viejo y Claudio Ptolomeo. Fontes Hispaniae antiquae, VII (1987) Barcelona: Instituto de Arqueología y Prehistoria.

53. Sabbioni, C.; Bonazza, A. y Zappia, G. (2002): Damage on hydraulic mortars: the Venice Arsenal. En Journal of Cultural Heritage, 3 (pp. 83-88). Elsevier.

54. Stylow, A. U. (1990). Neue Inschriften aus Carpetanien (Hispania Citerior). En Chiron, 20 (pp. 307-344). DAI. 
55. TIR (1993). Tabula Imperii Romani. Hoja K-30 (Caesaraugusta, Clunia). Madrid.

56. Urbano, A. (2005). Informe de actuación arqueológica en S.A.U. 5 ( $3^{a}$ fase): control de los movimientos de tierras. Inédito.

57. Urbano, A. (2010). Informe de actuación arqueológica, calle Real ,5 de Villamanta Inédito.

58. Velosa, A.L.; Coroado, J.; Veiga, M.R. y Rocha, F. (2007): Characterisation of roman mortars from Conímbriga with respect to their repair. En Materials Characterization. 10, 58 (pp. 1208-1216). Elsevier.

59. Zarzalejos, M. (2002). El alfar romano de Villamanta (Madrid). Madrid: Universidad Autónoma de Madrid.

60. Zendri, E.; Lucchini, V.; Biscontin, G. y Morabito, Z.M. (2004): Interaction between clay and lime in "cocciopesto" mortars: a study by 29Si MAS spectroscopy. Applied Clay Science, 25, 1-7. Paisley, Scotland. En Materials Characterization, A, 58, 11-12 (pp. 12081216). Elsevier Science. 